\title{
Energy Measurement in the T2K Oscillation Analysis
}

\section{Susan Cartwright ${ }^{* \dagger}$}

University Of Sheffield

E-mail: s.cartwright@shef.ac.uk

\begin{abstract}
A well-understood energy measurement is essential to control systematic errors in oscillation analyses, even in narrow-band off-axis experiments such as T2K. This contribution describes recent progress in energy reconstruction and $\pi^{0}$ rejection in the T2K far detector SuperKamiokande, together with techniques used for monitoring calibration, water quality and detector stability. Event reconstruction in the near detector ND280, essential for constraining the unoscillated neutrino flux, is also briefly discussed.
\end{abstract}

16th International Workshop on Neutrino Factories and Future Neutrino Beam Facilities - NUFACT2014, 25 -30 August, 2014

University of Glasgow, United Kingdom

\footnotetext{
*Speaker.

$\dagger$ on behalf of the T2K Collaboration.
} 


\section{Introduction}

The T2K experiment [1] is a long-baseline neutrino oscillation experiment in Japan. The neutrino beam is produced using protons from the J-PARC $30 \mathrm{GeV}$ proton storage ring incident on a graphite target and passes through a suite of near detectors before being observed by the SuperKamiokande experiment at a distance of $295 \mathrm{~km}$. Owing to an off-axis angle of $2.5^{\circ}$, the neutrino spectrum observed by Super-Kamiokande and the off-axis near detector ND280 is a narrow-band beam with a peak energy of approximately $600 \mathrm{MeV}$. The $v_{e}$ contamination in this $v_{\mu}$ beam originates principally from muon and kaon decays and has a somewhat broader spectrum [2], so good energy reconstruction is a vital ingredient in the control of systematic errors.

\section{Energy reconstruction in Super-Kamiokande}

Super-Kamiokande is a $50 \mathrm{kton}$ water Cherenkov detector, $39.3 \mathrm{~m}$ in diameter and $41.4 \mathrm{~m}$ high, instrumented with 1112920 " photomultiplier tubes (PMTs). It has a long and distinguished history as a stand-alone experiment, and $\mathrm{T} 2 \mathrm{~K}$ has benefited from the extensive experience of colleagues from Super-Kamiokande in optimizing reconstruction and understanding calibration and systematics. Nevertheless, a new fitting procedure developed by T2K has provided useful improvements in energy reconstruction and $\pi^{0}$ rejection for oscillation measurements.

Energy, or more precisely momentum, reconstruction in Super-Kamiokande is an integral part of the event reconstruction process. The input data comprise the charge and timing information from each PMT, together with known detector properties such as PMT response, water transparency, etc. For a single-ring event, the output data are the vertex position $(x, y, z, t)$, the particle ID $(e, \mu, \pi)$ and the particle momentum $p$. The standard Super-Kamiokande event reconstruction [3] is a sequential process consisting of the following stages:

1. find event vertex from timing information;

2. use Hough transform to find and count Cherenkov rings;

3. identify particle type using probability density function (pdf) for PMT charge information based on expected shape;

4. refit Cherenkov rings taking particle identification into account, and calculate momentum based on total number of photelectrons in a $70^{\circ}$ half-angle cone aligned along particle direction, corrected for attenuation and PMT acceptance.

This procedure yields a momentum resolution estimated at $(0.6+2.6 / \sqrt{p(\mathrm{GeV} / c)}) \%$ for singlering electrons and $(1.7+0.7 / \sqrt{p(\mathrm{GeV} / c)}) \%$ for muons.

The new method [4], based on a formalism developed for MiniBooNE [5], is a single-step maximum likelihood fit. For a single-ring fit, the likelihood is given by

$$
\mathscr{L}(\mathbf{x})=\prod_{i}^{\text {unhit }} P(i \text { unhit } \mid \mathbf{x}) \times \prod_{i}^{\text {hit }} P(i \text { hit } \mid \mathbf{x}) f_{q}\left(q_{i} \mid \mathbf{x}\right) f_{t}\left(t_{i} \mid \mathbf{x}\right)
$$


where the vector $\mathbf{x}$ contains the vertex position $(x, y, z, t)$, the track momentum $p$ and the track direction $(\theta, \phi), P(i$ hit/unhit $\mid \mathbf{x})$ is the probability that PMT $i$ will or will not register a hit, given track parameters $\mathbf{x}$, and $f_{q}$ and $f_{t}$ are the charge and timing pdfs for hit PMTs. Direct and scattered light are dealt with separately when calculating the probability density functions. The charge pdf $f_{q}$ is factorized into the predicted charge distribution $\mu_{i}$, which is a function of the particle momentum, direction and identity $(\mathrm{e}, \mu, \pi)$, and the PMT response. The particle identification information is contained within $f_{q}$, and a likelihood ratio is used to determine the most probable identification.
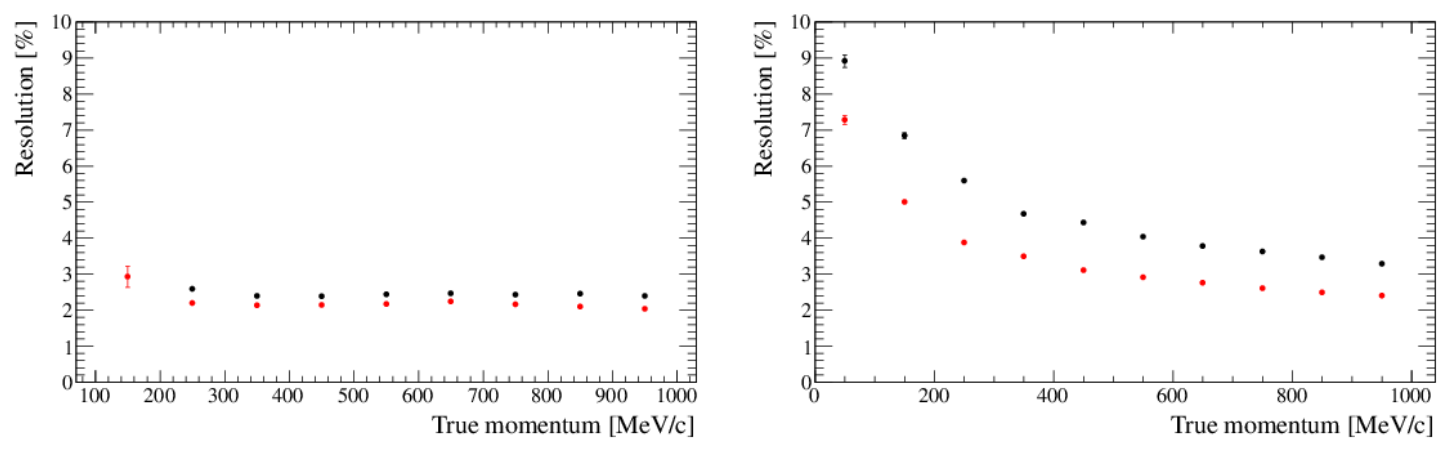

Figure 1: Momentum resolution for muons (left) and electrons (right), from particle-gun simulations. The standard fit results are in black and the results of the new fitter in red. (In comparing this plot to the momentum resulution figures quoted in the text, note that the quoted figures relate to Super-Kamiokande I, which had different readout electronics.)

The new fitter significantly improves the momentum resolution for electrons, as shown in figure 1. Its principal contribution to oscillation results to date, however, has been in reducing the $\pi^{0}$ background to $v_{e}$ appearance measurements.

Photons and electrons are indistinguishable in a water Cherenkov, and neutral-current $\pi^{0}$ production where one of the decay photons is missed is therefore a significant background in $v_{e}$ appearance. The new fitter incorporates a dedicated $\pi^{0}$ fit with 12 parameters: the vertex, the momenta and directions of the two photons, and the conversion length (distance from vertex to track start) for each photon. The fit proceeds by adding the expected charge distribution for a $50 \mathrm{MeV}$ electron (typical of the softer photon from a $\pi^{0}$ decay in $\mathrm{T} 2 \mathrm{~K}$ ) to a single-ring electron fit. This is scanned over all possible directions, and the direction with the best likelihood selected. A prefit is then performed, fixing all variables except the momenta. Finally, a full 12-parameter fit is performed using the predetermined direction and momentum as seed values.

The performance of this $\pi^{0}$ fitter is much superior to the standard Super-Kamiokande method, in particular greatly reducing the low mass tail in the reconstructed $\pi^{0}$ mass, as shown in figure 2 . This has resulted in a new criterion for $v_{e} / \pi^{0}$ separation, based on the reconstructed $\pi^{0}$ mass and the $\pi^{0} /$ electron likelihood ratio, as shown in the right panel of the figure. The new selection rejects $75 \%$ more $\pi^{0}$ background with no loss in signal efficiency; as the $\pi^{0}$ production cross-section has a large uncertainty, this represents a significant reduction in overall systematic error.

\section{Calibration and monitoring}

To minimize systematic errors in oscillation measurements, it is essential that the detector is 

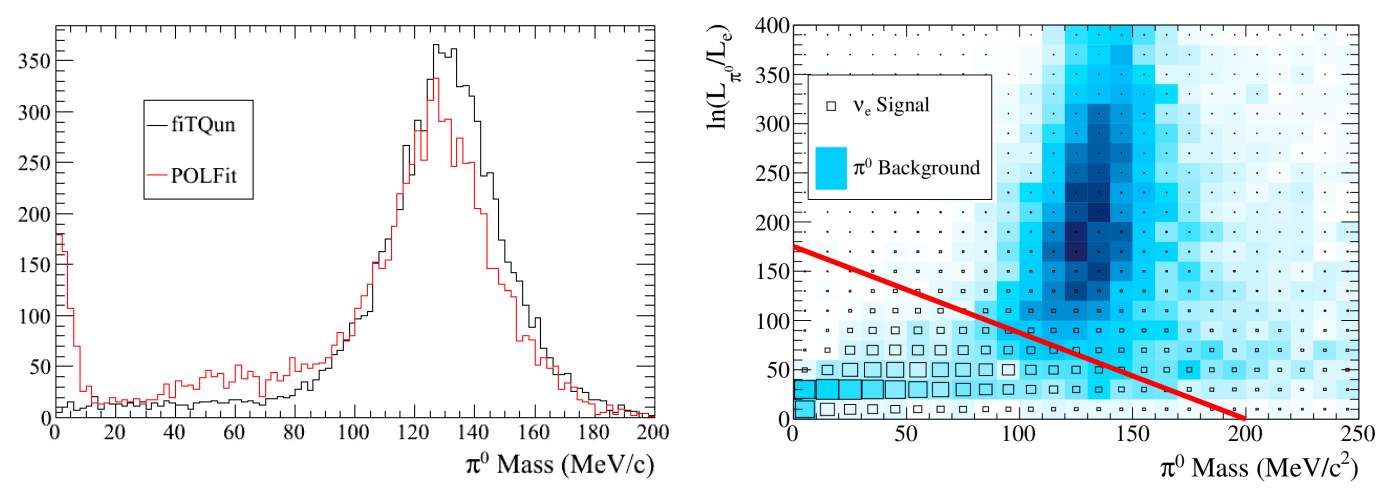

Figure 2: Two-photon invariant mass (left), reconstructed using the standard fitter POLFit (red) and the new fitter FiTQun (black). The right panel shows the $\pi^{0}$ rejection cut using the new fitter, as applied in [6]: the blue shading is the expected $\pi^{0}$ background, and the unfilled boxes are the $v_{e}$ signal. Only events below the red line are retained.

effectively calibrated and that its operational stability is well monitored. In both these aspects, T2K gains from the fact that Super-Kamiokande has operated and continues to operate as a stand-alone experiment: in particular, most routine monitoring is done using atmospheric muons.

The hardware calibration of Super-Kamiokande is discussed in detail in [7]. In brief:

- The PMT relative gain and timing are monitored using a stable light source permanently deployed in the centre of the water tank. The light source is a pulsed nitrogen-driven dye laser equipped with a diffuser ball to produce an isotropic light distribution. The wavelength of the light is $398 \mathrm{~nm}$ and the pulse duration is $0.2 \mathrm{~ns}$.

- Absolute gain calibration is achieved using $\gamma$-rays of around $9 \mathrm{MeV}$ energy from the reaction ${ }^{58} \mathrm{Ni}(\mathrm{n}, \gamma){ }^{59} \mathrm{Ni}$, with the neutrons supplied by a ${ }^{252} \mathrm{Cf}$ source. When deployed at the centre of the tank, the source produces an average of 0.004 photoelectrons per PMT per event, which ensures that almost all recorded PMT signals correspond to single photoelectron events. This calibration was carried out at the beginnings of Super-Kamiokande phases III and IV.

- Light absorption and scattering in water are monitored by shining collimated laser light $(\lambda \lambda$ $337,375,405,445$ and $473 \mathrm{~nm}$ ) vertically downward from the top of the tank and analysing the response from the PMTs.

The stability of the detector performance is monitored using atmospheric muons. The overall trigger efficiency is checked by recording the average daily number of fully-contained atmospheric muon events in the Super-Kamiokande fiducial volume, averaged over successive two-month periods. The energy scale is monitored using stopping muons, identified by the presence of a second, electron-like, ring from the decay electron: the range of the muon is compared with its reconstructed momentum, and the reconstructed momentum of the decay electron is compared with expectations from the decay kinematics. All of these stability indicators are found to be constant to within $\pm 1 \%$.

These cosmic-ray data are also used to estimate the energy scale systematic error for SuperKamiokande in $\mathrm{T} 2 \mathrm{~K}$ oscillation analyses[6]. The estimated scale error of $2.3 \%$ is based on the 
comparison of reconstructed momentum and range for stopping muons with energies comparable to the T2K neutrino beam, the distribution of reconstructed momenta of decay electrons from stopping muons, and the reconstructed mass of $\pi^{0}$ candidates in atmospheric neutrino interactions.

\section{Systematic errors in energy reconstruction}

In addition to detector performance, there are theoretical uncertainties that can affect the interaction kinematics and hence bias the reconstructed energy.

Final-state interactions involving multiple nucleons within the target nucleus can affect the kinematics of the outgoing lepton. Nucleons exiting the nucleus will be below Cherenkov threshold in Super-Kamiokande, and hence such interactions cannot be distinguished from pure quasi-elastic scattering (CCQE). In such events, the neutrino energy estimated from the two-body kinematics of CCQE interactions is an underestimate of the true energy. Various theoretical models of multinucleon interactions are available; the default used in the NEUT Monte Carlo [9] is pionless $\Delta$ decay. In the T2K $v_{\mu}$ disappearance analysis [8], this was compared with the more sophisticated model of Nieves et al. [10]: although the predictions of the two models differ significantly, multiple trials with fake data sets showed that the mean expected bias on the oscillation parameters caused by this difference was $<1 \%$.

Secondary interactions of pions in the Super-Kamiokande detector volume can also affect the event classification and reconstructed neutrino energy. Charge exchange scattering of $\pi^{+}$to $\pi^{0}$ provides an additional contribution to the $v_{e}$ appearance background, though this is effectively reduced by the new $\pi^{0}$ fit described above. Absorption of a $\pi^{+}$after a path length short enough to produce no detectable Cherenkov signature will cause a charged-current event with a pion to be misclassified as CCQE with a lower neutrino energy, and elastic and quasi-elastic scattering of a pion can cause it to be misidentified or mismeasured. These effects are studied by modifying the pion interaction parameters in NEUT, and contribute a systematic error of order $1 \%$.

\section{The T2K near detectors}

The T2K near detector suite monitors the unoscillated beam, including the flux, direction, energy spectrum and intrinsic $v_{e}$ content, and also measures neutrino interaction cross-sections relevant to oscillation analyses. The near detector constraints on the neutrino flux are crucial to the oscillation measurements: for example, without these constraints, the beam flux systematic error in the $v_{e}$ appearance measurement reported in [6] would increase from $2.9 \%$ to $25.9 \%$.

The near detector suite consists of two separate detectors: the on-axis detector INGRID [1], which is centred on the nominal beam direction, monitors the beam profile, the beam direction, and the rate of neutrino interactions per proton on target, while the off-axis detector ND280 is situated at the same off-axis angle as Super-Kamiokande and measures the properties of the off-axis beam.

Monitoring the beam direction is critical for an off-axis experiment. A $1 \mathrm{mrad}$ change in beam direction results in a $2-3 \%$ change in the energy scale, which is comparable to the $2.3 \%$ energy scale error from Super-Kamiokande. INGRID has a cruciform cross-section, to measure the vertical and horizontal beam profile, and is complemented by a muon monitor located immediately downstream of the beam dump in the T2K beamline. The results from INGRID show that the beam 
position has been stable to within $\pm 0.3 \mathrm{mrad}$ throughout $\mathrm{T} 2 \mathrm{~K}$ running, and to within $\pm 0.1 \mathrm{mrad}$ for most of that period. The neutrino interaction rate is stable to better than $0.7 \%$ throughout.

The off-axis detector, ND280 [1], is based on the former UA1/NOMAD dipole magnet, and consists of two main sections: a scintillator-based $\pi^{0}$ detector, or P0D, and a tracker consisting of three TPCs interleaved with two scintillator-based Fine-Grained Detectors (FGDs) to provide an active target. Both sections are surrounded by an electromagnetic calorimeter or ECal, and the magnet yoke is instrumented with muon detectors.

The ND280 analyses used to constrain the oscillation fits are based on the tracker region of the detector. Track momenta are measured using track curvature in the TPCs, and particle identification is accomplished using $d E / d x$, supplemented by ECal shower measurements where appropriate. Charged-current events are identified by the presence of a muon and divided into three categories, $\mathrm{CC}-0 \pi, \mathrm{CC}-1 \pi^{+}$and $\mathrm{CC}$-other, based on the number of reconstructed pions in the final state (respectively none, exactly one $\pi^{+}$, and anything else). These samples are then fitted to derive the beam flux parameters and common cross-sections, which are propagated to Super-Kamiokande. The principal systematic errors in this procedure do not involve the energy reconstruction, which is straightforward, but are dominated by out-of-fiducial-volume events with misreconstructed vertices and final-state pion reinteractions causing migration between the event categories. In addition to this flux monitoring, a recent analysis [11] measured the intrinsic $v_{e}$ content of the off-axis beam, obtaining a result of $1.01 \pm 0.10$ times the prediction from the beam simulation [2]. This is an important independent confirmation of this quantity, which of course represents an irreducible background to the $v_{e}$ appearance measurement.

\section{Summary}

T2K benefits from a well-understood far detector with mature calibration, reconstruction and analysis tools. Despite this, performance is still being improved, most recently by the development of a new reconstruction method which has substantially reduced the $\pi^{0}$ background—and its associated systematic error-in $v_{e}$ appearance measurements.

Inputs from the near detectors, in particular the off-axis detector ND280, are crucial in monitoring the beam direction (and hence the energy scale) and constraining the unoscillated flux and cross-section models. These constraints reduce the flux-associated systematic error budget in T2K oscillation analyses by about a factor of 10 , from $25.9 \%$ to $2.9 \%$ in [6].

The continuing use of Super-Kamiokande as an atmospheric neutrino detector is also advantageous, as the atmospheric muon sample is used to monitor the stability of the detector and the absolute energy scale.

As a result of steady improvement in all aspects of the analysis, the systematic error budget in $\mathrm{T} 2 \mathrm{~K}$ oscillation measurements has steadily decreased, and is now a factor of 2 smaller than it was in the first oscillation analysis reported in 2011. There is no single dominant systematic error in the current error budget, see for example [6] and [8], but continued incremental improvements are expected in both reconstruction and theoretical aspects, e.g. cross-section modelling. 


\section{References}

[1] K Abe et al. (T2K Collaboration), The T2K experiment, Nucl. Instrum. Meth. A 659 (2011) 106-135 [arXiv:1106.1238 [physics.ins-det]].

[2] K Abe et al. (T2K Collaboration), The T2K neutrino flux prediction, Phys. Rev. D 87 (2013) 012001 [arXiv:1211.0469 [hep-ex]].

[3] Y Ashie et al. (Super-Kamiokande Collaboration), Measurement of atmospheric neutrino oscillation parameters by Super-Kamiokande I, Phys. Rev. D 71 (2005) 11205 [hep-ex/ 0501064 ].

[4] M Wilking, A new method for event reconstruction in large Cherenkov detectors, talk given at New Directions in Neutrino Physics, Aspen, Colorado (2013); available at https://indico.cern.ch/event/224351/contribution/8/material/slides/0.pdf

[5] RB Patterson et al., The extended-track reconstruction for MiniBooNE, Nucl. Instrum. Meth. A 608 (2009) 206-244 [arXiv:0902.2222 [hep-ex]].

[6] K Abe et al. (T2K Collaboration), Evidence of electron neutrino appearance in a muon neutrino beam, Phys. Rev. D 88 (2013) 032002 [arXiv:1304.0841 [hep-ex]].

[7] K Abe et al. (Super-Kamiokande Collaboration), Calibration of the Super-Kamiokande Detector, Nucl. Instrum. Meth. A 737 (2014) 253-272 [arXiv:1307.0162 [physics.ins-det]].

[8] $\mathrm{K}$ Abe et al. (T2K Collaboration), Precise measurement of the neutrino mixing parameter $\theta_{23}$ from muon neutrino disappearance in an off-axis beam, Phys. Rev. Lett. 112 (2014) 181801 [arXiv:1403.1532 [hep-ex]].

[9] Y Hayato, A neutrino interaction simulation program library NEUT, Acta Physica Polonica B 40 (2009) 2477-2489.

[10] J Nieves, IR Simo, and M Vicente Vacas, The nucleon axial mass and the MiniBooNE quasielastic neutrino-nucleus scattering problem, Phys. Lett. B 707 (2012) 72-75 [arXiv:1106.5374 [hep-ph] ].

[11] K Abe et al. (T2K Collaboration), Measurement of the intrinsic electron neutrino component in the T2K neutrino beam with the ND280 detector, Phys. Rev. D 89 (2014) 092003 [arXiv: 1403.2552 [hep-ex]]. 